\title{
Presentación de un caso de queratitis por Acanthamoeba, su aislamiento clínico y tratamiento exitoso con antisépticos en el departamento del Quindío, y revisión de la literatura.
}

\section{Case report of Acanthamoeba keratitis, its clinical isolation and successful treatment with antiseptics in the department of Quindio; and its literature review. Short Title: A case of keratitis by Acanthamoeba in Quindio.}

\author{
Jhon Carlos Castaño-Osorio* \\ Recibido: Agosto 3 de 2009 \\ Aceptado: Noviembre 9 de 2009 \\ Correspondencia:Maestria en Ciencias Biomédicas. Universidad del Quindío. Carrera 15 calle 12 norte, Armenia. Email: yiyuma@yahoo.com; \\ jhoncarlos@uniquindio.edu.co
}

\section{Resumen.}

La queratitis por Acanthamoeba es una entidad clínica cuya incidencia se ha venido incrementando recientemente, debido al uso de lentes de contacto blandos. En el presente artículo se presenta un caso clínico de una joven usuaria de este tipo de lentes quien desarrolló un cuadro de queratitis que se diagnosticó inicialmente como una queratitis herpética. Al no responder a tratamiento con trivirina (trifluridina, viromidin 1\%) y aciclovir (3\%) se consideró el diagnóstico de queratitis micótica, aunque sin respuesta al tratamiento con anfotericina B. El diagnóstico definitivo de queratitis por Acanthamoeba se logró establecer por observación microscópica directa de de trofozoítos de Acanthamoeba spp en un raspado corneano. La presencia de este microorganismo se confirmó mediante la obtención de abundantes trofozoítos móviles en un cultivo de células Vero. Luego de instaurar el tratamiento con digluconato de clorohexidina y polihexametil binguadina se logró una rápida mejoría clínica. Este hallazgo se constituye en el primer caso de queratitis por Acanthamoeba spp. en el Departamento del Quindío y en el primer reporte de tratamiento médico exitoso con estos dos productos. Es de anotar que en presente caso el diagnóstico etiológico se dio a los dos meses de inicio de la sintomatología) debido en parte a que la queratitis por Acanthamoeba spp. puede simular otras entidades infecciosas de carácter viral como el herpes o micótico producidas por Candida, Aspergillus y Fusarium, y que por la poca frecuencia de la entidad, los oftalmólogos no piensan en esta posibilidad diagnóstica.

Palabras Claves: Queratitis, Acanthamoeba, Lentes de contacto, Digliconato de clorohexidina, Polihexametil binguadina.

\begin{abstract}
Keratitis by Acanthamoeba is a clinical condition whose incidence has been increasing recently due to the use of soft contact lenses. This article presents a case of a young user of such lenses who developed a cadre of keratitis initially diagnosed as herpetic keratitis. By not responding to treatment, it was determined as a definitive diagnosis of Acanthamoeba keratitis after establishing by direct microscopic observation of trophozoites of Acanthamoeba corneal scraping. The presence of this microorganism was confirmed by obtaining abundant motile trophozoites in Vero cell culture. After initiation of therapy with chlorhexidine digluconate and polyhexamethylene biguadine, a rapid clinical improvement was achieved. This finding constitutes the first case of Acanthamoeba keratitis in the Department of Quindio and the first report of successful medical treatment with these two products. It should be noted that in this case the etiologic diagnosis was two months after the onset of symptoms in part due to Acanthamoeba keratitis may look the same to other conditions by different infectious agents such as herpes viral or fungal. Due to the rarity of Acanthamoeba, ophthalmologists do not consider this diagnostic possibility. Key words: Keratitis, Acanthamoeba, Contact lens chlorhexidine digluconate, polyhexamethylene binguadie.
\end{abstract}

*Programa de Medicina. Facultad Ciencias de la Salud. Universidad del Quindío 


\section{INTRODUCCIÓN}

$\mathrm{E}$ I género Acanthamoeba comprende protozoos ubicuos de vida libre capaces de causar infecciones en humanos, incluyendo encefalitis, queratitis y neumonía. Acanthamoeba spp. es el protozoo mas prevalente en el ambiente. Se ha aislado del suelo, polvo, aire, agua natural o tratada, agua de mar, agua de piscinas, aguas residuales, sedimentos, unidades de aire acondicionado, llaves de agua domestica, plantas de tratamiento de agua, botellas de agua, unidades dentales, unidades de diálisis de hospitales, en estaciones de lavado de ojos, en lentes de contacto y en los empaques de los lentes de contacto; así como contaminantes de cultivo de bacterias, hongos y células de mamíferos y presenta una amplia distribución mundial (1-3).

\section{Queratitis por Acanthamoeba spp:}

La infección ocular causada por amibas de vida libre produce un cuadro inespecífico de queratoconjuntivitis y ulceraciones de la cornea, de evolución tórpida y de difícil manejo, que fácilmente puede terminar en perforación ocular y endoftalmitis si no se hace un diagnóstico temprano. Las manifestaciones clínicas de la enfermedad pueden confundirse con las de otras entidades como la infección por herpes simplex (seudo dendrítica con estroma en forma de anillo) y otros tipos de queratitis, lo que dificulta el diagnóstico diferencial, retarda el tratamiento y por lo tanto empeora el pronóstico $(4,5)$.

La primera descripción de la queratitis por Acanthamoeba spp. fue realizada por Nagington en 1974 (6). A partir de la década del 80 los números de casos aumentaron significativamente.

El $85 \%$ de los aislamientos de Acanthamoeba spp se obtuvieron por cultivos de biopsia de córnea de pacientes que utilizaban lentes de contacto blandos o semi-blandos. La mayoría de los casos informados están asociados a una incorrecta limpieza de los lentes de contacto (4).

Visvesvara (1975) realizó una caracterización biológica de los trofozoítos de $A$. polyphaga aislados de los raspados cornéanos, en infecciones experimentales in vitro en células Vero así como la determinación de su patogenicidad en ratones BALB/C . Los efectos citopáticos (CPE) observados corresponden a encogimiento celular, picnocitosis nuclear y discontinuidad de la superficie celular a los 5 días del cultivo y una destrucción total del cultivo celular entre los 8-10 días post-infección. Cuando se instilaron los trofozoítos de la ameba en los ratones BALB/c por vía intranasal, solo uno de veinte ratones murió a los 28 días postinoculación (7).
En el Japon Zheng y col. (2004) determinaron que tanto los trofozoítos de A. castellani (cepa de referencia ATCC 30868) como un lisado de los mismos son capaces de inducir apoptosis en células epiteliales cornéanas (Línea celular HCE2) luego de 12 horas de exposición, proponiendo este mecanismos como el responsable del daño del epitelio cornéano característico de la queratitis por Acanthamoeba $\operatorname{spp}(8)$.

Bottone y col. (1995) observaron que la contaminación de los sistemas de cuidado de los lentes de contacto con Acanthamoeba spp. y con especies bacterianas capaces de soportar el crecimiento amebiano pueden ser el primer paso en la patogénesis de la queratitis inducida por Acanthamoeba spp, al proporcionar un inoculo grande de amebas (9).

\section{Factores de riesgo:}

Entre los factores predisponentes a las queratitis se destacan los traumatismos cornéales, el contacto con cuerpos extraños o la exposición al agua templada (de una bañera o piscina, por ejemplo); pero el factor de riesgo más importante para contraer esta infección es el uso de lentes de contacto, sobre todo si se usan soluciones salinas caseras o agua corriente para el lavado de las mismas, el no desinfectarlas apropiadamente o con la frecuencia recomendada y la utilización de los lentes durante la práctica de la natación (9).

Aunque el uso de lentes de contacto es el factor pre disponente más frecuentemente asociado a la queratitis por Acanthamoeba spp, la incidencia de la enfermedad entre los usuarios de este tipo de lentes sigue siendo muy baja, lo que indica que éste microorganismo es relativamente, poco virulento, que existe inmunidad innata en el huésped y que el epitelio corneal ofrece una barrera frente a la penetración de las amebas en el estroma y el desarrollo consiguiente de queratitis. En los pacientes con antecedentes de traumatismo, las amebas pueden llegar directamente al estroma, y en usuarios de lentes de contacto dicho acceso se facilita por microtraumatismos repetidos, aunque se ha visto que $A$. castellanii es capaz de atravisar el epitelio intacto de la córnea (7).

Aunque no están bien caracterizados los mecanismos del sistema inmunitario que operan contra Acanthamoeba spp, en estudios experimentales en ratones BALB/c se ha visto que los neutrófilos y macrófagos son responsables de la respuesta inflamatoria celular observada en el área de la infección. Parece que los macrófagos conjuntivales tienen un papel muy importante en la primera línea de defensa y en la eliminación de los trofozoítos, ya que su desaparición 
aumenta la incidencia de la infección, agrava la enfermedad y determina que la queratitis se inicie más temprano y tenga un curso más prolongado (6-8).

Si bien el primer caso de queratitis por Acanthamoeba spp. (QA) se describió en 1974, y hasta 1984 se mantuvo como una enfermedad muy poco frecuente, en la mayoría de casos asociada a traumatismos de la córnea y a la exposición a aguas contaminadas, a mediados de los años ochenta, aumentó de forma espectacular el número de casos y se atribuyó al incremento en el uso de lentes de contacto y a su incorrecta manipulación y desinfección (6,7,9-11).

A pesar de que la QA es rara, con una incidencia anual en los Estados Unidos entre 1,65 y 2,01 casos por millón de usuarios de lentes de contacto, puede ser 15 veces más frecuente en el Reino Unido, en Europa y en Hong Kong (4). Recientemente, se ha visto un incremento en el número de casos de QA en los Estados Unidos, atribuibles a los cambios de factores clínicos y demográficos como el uso cada vez más frecuente de lentes de contacto blandos en adolescentes, patogenicidad del organismo, cambios en la higiene de los lentes y a los cambio de las normas para las fuentes de abastecimiento de agua (4). Los lentes de contacto blandos ( $\mathrm{SCL}$ ) siguen siendo el principal factor de riesgo para la $Q A$, aunque el trauma corneal seguido de la exposición a Acanthamoeba spp por si solo puede incitar a la queratitis $(7,10,12-14)$.

\section{Epidemiología:}

Manikandan y col., (2004) reportaron en un incremento de la incidencia de QA no asociado con el uso de lentes de contacto, pero si con los traumas oculares con objetos sólidos en el sur de la India, un período de seis años. La QA en este país representa cerca del $1 \%$ de todos los casos de queratitis microbiana. La perdida visual severa en algunos de estos pacientes ocurrió debido a un diagnóstico tardío o a un diagnóstico errado y por un tratamiento antimicrobiano inapropiado (15).

\section{Manifestaciones Clínicas:}

La queratitis amebiana es la manifestación clínica más habitual de la infestación por el género Acanthamoeba. La infección progresa originando una ulceración de la córnea y puede dar como resultado ceguera en casos muy graves, por lo que se caracteriza por ser dolorosa e invalidante. El cuadro clínico varía según el momento de la primera consulta. Al principio, las amebas se encuentran en el epitelio corneal, pero si la enfermedad progresa ocurre la invasión del estroma. En sus inicios, se caracteriza por limbitis, queratopatía punteada, infiltrados epiteliales, subepiteliales o perineurales (queratoneuritis radial). El paciente sufre enrojecimiento, lagrimeo, fotofobia y dolor de diversa intensidad, pero desproporcionado respecto a los signos oculares, así como visión borrosa $(12,13)$.

Si la enfermedad progresa, puede observarse ulceración, infiltrados anulares, placas endoteliales y uveítis anterior, con o sin hipopión (presencia de pus en la cámara anterior del ojo), y más infrecuentemente, edema corneal. Si el proceso se agrava, se pueden producir abscesos, escleritis, glaucoma, catarata e infección microbiana secundaria. Lo más característico es la presencia de un infiltrado anular compuesto por células inflamatorias (neutrófilos).Los pacientes que sufren esta infección son generalmente inmunocompetentes; no obstante, no desarrollan inmunidad protectora apreciable, por lo que es posible la reinfección $(6,12,14,16)$.

\section{Diagnóstico y Tratamiento:}

Es una enfermedad difícil de diagnosticar y tratar, ya que las manifestaciones clínicas se confunden a menudo con las de las queratitis herpética, fúngica o micobacteriana, lo que provoca que el diagnóstico correcto y el comienzo del tratamiento se retrasen $(12,16)$. Las limitaciones técnicas del diagnóstico pueden dar lugar a una demora en el diagnóstico o a un diagnóstico equivocado. A su vez, un retraso en el diagnóstico se asocia con un peor pronóstico visual y una evolución más prolongada $(15,16)$.

Para el diagnostico se requiere un examen de laboratorio simple y rápido consistente en la coloración con $\mathrm{KOH}$ y visualización microscópica de la muestra del exudado cornéano. El diagnóstico a su vez permite el inicio oportuno de un tratamiento antimicrobiano adecuado (16). El raspado corneal o la biopsia coloreadas con Giemsa, acido per yódico de Schiff (PAS), blanco de calcofluor, naranja de acridina o las improntas pueden revelar la presencia de quistes o trofozoítos, pero tienen el inconveniente de ser herramientas diagnosticas invasivas que añaden una carga adicional para el proceso de cicatrización corneal. Los cultivos utilizando agares no nutritivos suplementados con E.coli son igualmente útiles por su alta sensibilidad y especificidad, permitiendo la demostración de los trofozoítos(16).

El tratamiento de la QA es básicamente médico y se fundamenta en el uso de antiamebianos de alta eficiencia como las diamidinas (isetionato de propamidina, la hexamidina), que inhiben el consumo de oxigeno e inducen la salida de aminoácidos de la célula y el daño de la superficie celular; y las binguadinas (clorohexidina, polihexametil binguadina- $P H M B$ ) cuyo sitio primario de acción es la membrana citoplasmática, debido a la interacción electrostática con los fosfolípidos ácidos, siendo las binguadinas los medicamentos de primera elección, solas o asociadas a diamidinas (ambas son quisticidas). 
La efectividad de los tratamientos médicos utilizando estos medicamentos han sido variables, reportándose tasas de curación de las lesiones cornéales desde el $92 \%$ al $100 \%$ con mejoría notable de la agudeza visual, disminución del dolor regresión de la neuritis y de los infiltrados inflamatorios al final del tratamiento cuando se utilizan combinación de PHMB, propamidina, antibióticos de amplio espectro y antimicóticos como anfotericina B, Neomicina, clotrimazol. Sin embargo cuando estos compuestos se han utilizado como monoterapia los índices de curación disminuyen al 78\% con PHMB y de $85,7 \%$ con clorohexidina ( ). Los reportes de resistencia a estos medicamentos presentann resultados devastadores como la evolución hacia la perforación cornéana y perdida de la visión en el ojo comprometido debido a la opacidad corneal residual, debiendo recurrir a tratamiento quirúrgico (17-25).

La utilización de corticoides es controvertida debido a la asociación de su uso con fallas en el tratamiento antiamebiano y a la desarrollo precoz de recurrencias. Sin embargo o existen indicaciones en los casos de inflamación severa de la cornea o de la cámara anterior y para el manejo del dolor severo que no cede a los analgésicos $(13,17,18,24,26,27)$.

\section{Presentación del caso.}

Paciente de 20 años de edad, sexo femenino, con antecedentes de miopía en ambos ojos, usuaria de SLC desde hace 1 año, quien consultó al Centro de Investigaciones Biomédicas (CIBM) de la Universidad del Quindío el 24 de Octubre de 2006 por "dolor ocular y sensación de cuerpo extraño en ojo izquierdo", luego de cuadro clínico de 2 meses de evolución consistente en ojo rojo y sensación de ardor ocular por lo cual había consultado a múltiples especialistas (optómetra, $n=1$, oftalmólogo, $: n=6$ ) quienes ordenaron inicialmente oclusión ocular por 5 días sin mejoría, así como manejo con trivirina (trifluridina, viromidin $1 \%$ ) y aciclovir (3\%) tópico en ungüento ante la sospecha clínica de queratitis herpética: Puesto que no se presentó mejoría, se indicó cambiar la formulación del aciclovir tópico a sistémico y suspensión de la trivirina. Se adicionó un AINES con lo cual mejoró notablemente al mes de tratamiento.

A los 15 días el cuadro empeoró por lo que se inició aciclovir tópico, tobramicina $0.3 \%$ y dexametasona $0.1 \%$ por 7 días, presentando mejoría clínica. Sin embargo, 15 días después presentó nuevamente molestia ocular (visión de puntos) por lo que se consideró que el cuadro correspondía a una queratitis herpética y se indicó trivirina y aciclovir sistémico y tópico; a los 7 días presentó "sombra en la córnea" y consulta otro oftalmólogo quien diagnosticó queratitis micótica y dio tratamiento con anfotericina $B$ y aciclovir. La paciente presentó mejoría a los 5 días, pero 5 días después empeoró el cuadro clínico por lo que se indicó tratamiento con aciclovir en ungüento y anfotericina $B$, presentando mejoría clínica y disminución de la profundidad de la úlcera, a los 3 días.

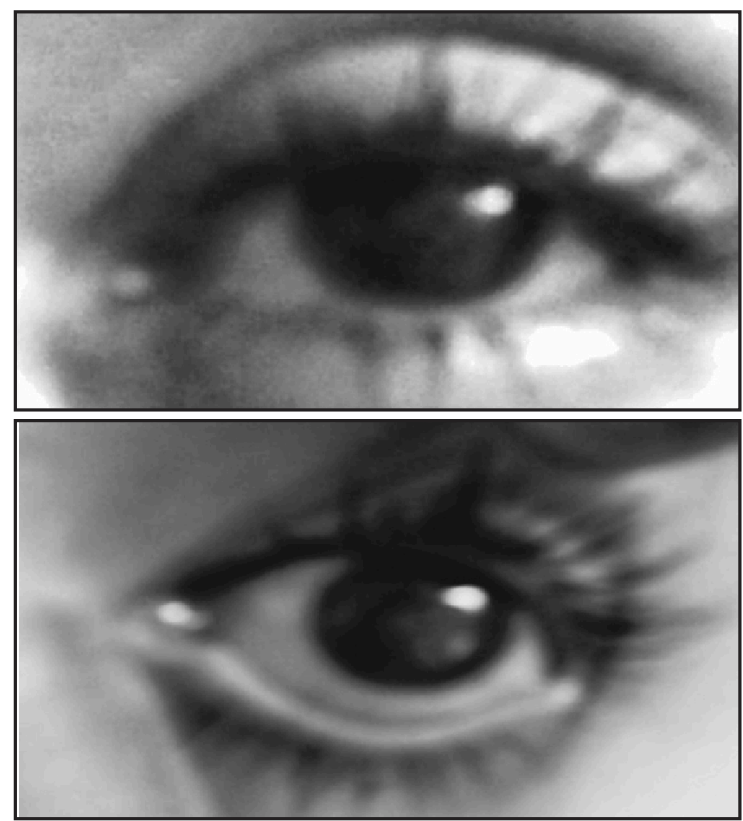

Figura 1:Registro fotográfico inicial, nótese la opacidad cornéana extensa

Pasados 15 días empeoró nuevamente y es referida al CIBM., donde se tomó muestra de raspado corneal, a partir de la cual en el examen directo bajo microscopio de luz se visualizaron múltiples microorganismos translúcidos de núcleo prominente y pseudópodos en forma de espinas así como quistes de doble pared y forma poligonal de aproximadamente 18 micras. Adicionalmente, se realizó inoculación de la muestra en cultivo de células Vero, evidenciando efecto citopático y proliferación de numerosos microorganismos móviles durante las primeras 24 horas pos inoculación.

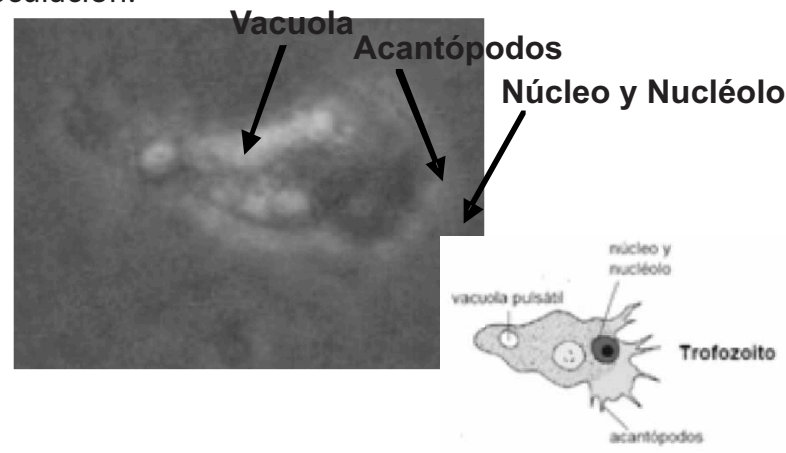

Figura 2: Trofozoitos de Acanthamoeba en cultivo .Microscopio invertido Olympus 40X Pasadas 72 horas se observa destrucción de toda la monocapa y presencia de microorganismos enquistados. 


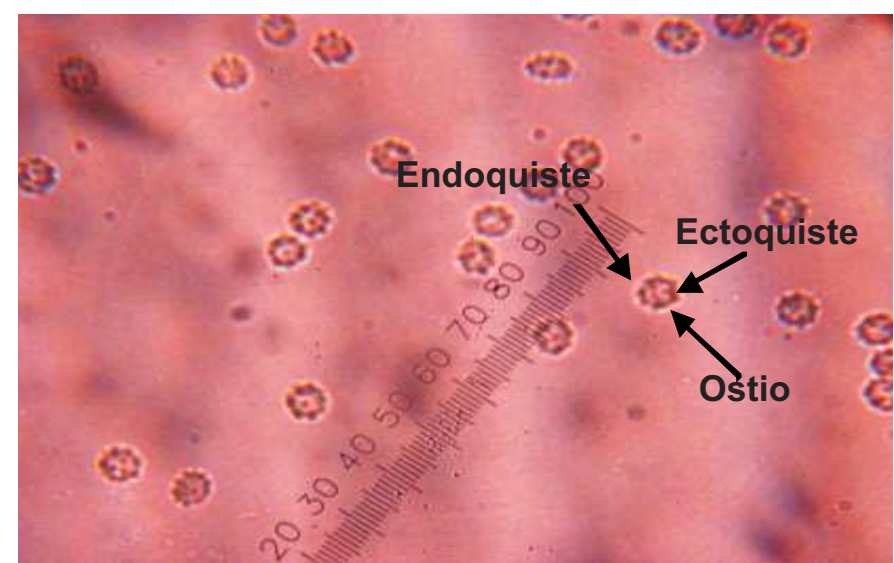

endoquiste

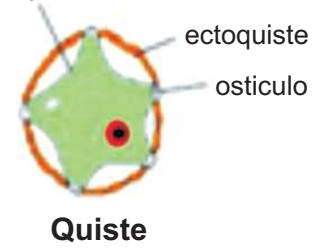

Figura 3. Quistes en cultivo. Nótese la forma poligonal y la espiculas en su superficie características Microscopio invertido Olympus $10 \mathrm{X}$

\section{A}

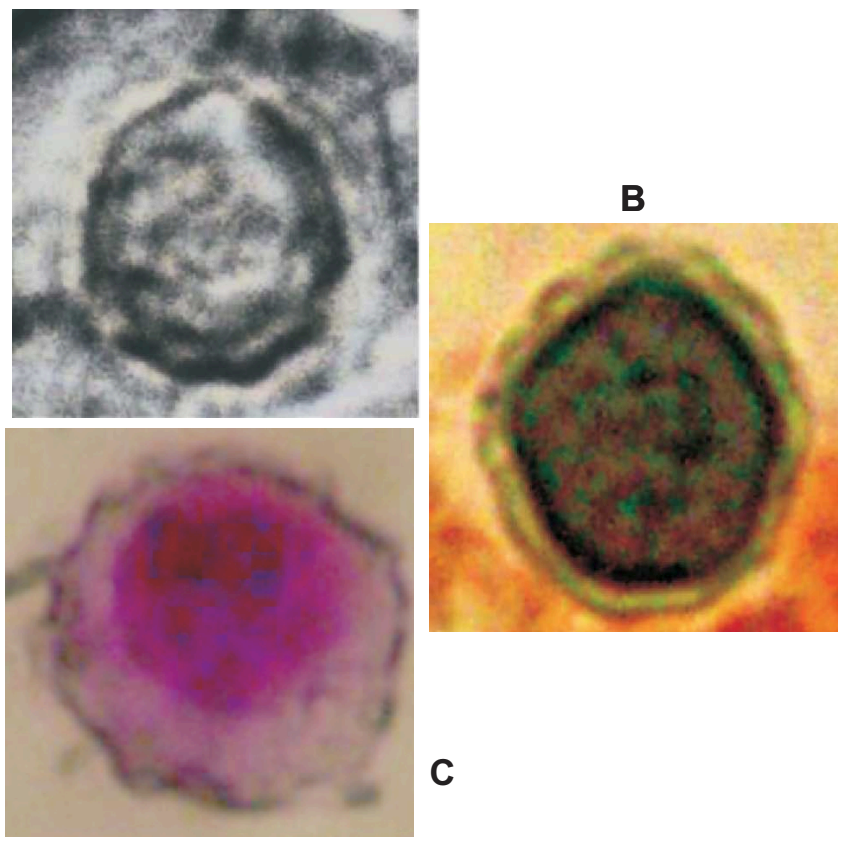

Figura 4. Quistes de Acanthamoeba spp observadas en microscopio óptico sin coloración (a), con coloración de

lugol parasitologico(b) y coloración de Giemsa(c) . Microscopio invertido Olympus 40X

Una vez establecido el diagnóstico de QAse inició tratamiento con digluconato de clorohexidina al 0,12\% ( Perioxidin ${ }^{\circledR}$ ) a una dosis de 1 gota en el ojo afectado cada 30 minutos por 7 días y 1 gota cada 2 horas durante 7 días más. $A$ los 15 día se inició tratamiento con Polihexametil binguadina(PHMB) al 0,02\% una gota en el ojo afectado cada 6 horas. Con este esquema se logro cicatrización completa de la úlcera cornéana a los 15 días.

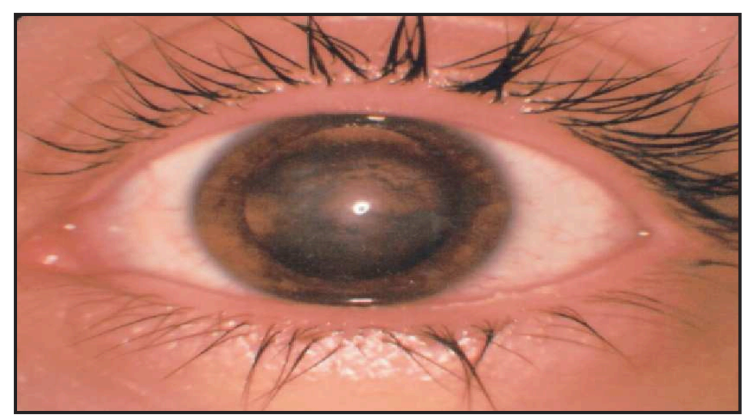

Figura 5. Registro de control 10 meses después de tratamiento, observe la mejoría de la superficie cornéana, y la presencia de neovascularización.

Al presente, la paciente continua recibiendo este esquema terapéutico (18 meses) sin presentar recaídas; su agudeza visual mejoró notablemente de visión de bultos inicial a 20/100; sin embargo, su cornea quedó con una opacidad residual y una neovascularización marginal por lo que está a la espera la realización de una queratoplastia.

\section{Discusión.}

Se presenta un caso de QA en una mujer joven usuaria de $\mathrm{SCL}$, cuyo diagnóstico se realizo después de 2 meses del iniciado los síntomas, y quien recibió múltiples tratamientos antivirales y antimicóticos acompañado de antiinflamatorios esteroideos y no esteroideos.

Al igual que en otros casos publicados de QA, en el presente caso la queratitis herpética es el diagnóstico erróneo más descrito en la literatura, siendo a su vez la causa más frecuente que conduce al diagnóstico tardío de la QA (25).E tratamiento adecuado con cationes anionicos ( $P M H B$ y digluconato de clorhexidina) permitió la rápida cicatrización de la lesión. Puesto que estos compuestos no están disponibles en el mercado para el uso terapéutico, se utilizó el colutorio Perioxidin ${ }^{\circledR}$ cuya formulación es a base de digluconato de clorohexidina, polihexametil binguadina utilizada para el tratamiento químico de piscinas libre de cloro La mezcla se preparó en solución salina fisiológica estéril.

El diagnóstico se logro establecer por la observación microscópica directa de un raspado cornéano en el cual se visualizaron trofozoítos de Acanthamoeba spp. y se confirmó luego la presencia de este microorganismo con la obtención de abundantes trofozoítos móviles en un cultivo de células Vero y el posterior desarrollo de los quistes característicos 
con doble pared y forma poligonal. Si consideramos el comportamiento en el cultivo celular de este aislado al compararlo con lo reportado por Visvesvara y col, (1975) quienes observaron los efectos citopáticos en el cultivo celular al cabo de 5 días de incubación, podemos considerar que el aislado obtenido en este caso presenta una alta virulencia pues se observaron efectos citopaticos desde las 24 horas de inoculación con una destrucción total de la monocapa en solo 72 horas postinoculación.

En la literatura médica Colombiana consultada, Rosero en 2001 reporta una incidencia promedio de 23 casos por año de queratitis por hongos en el Valle del Cauca, ninguno de ellos conifectado por Acanthamoeba spp(28), De la Torre en el 2003 reportó una serie de 18 casos de queratitis infecciosas en el servicio de oftalmología del Hospital Universitario del Valle, de los cuales ninguna se asoció a Acanthamoeba spp como agente causal (29) Igualmente en la revista electrónica de la clínica Barraquer de Bogotá no aparecen casos clínicos ni reportes de la incidencia de esta entidad; sin embargo, Serrano y col. (AÑO) realizaron un estudio prospectivo de pacientes con diagnóstico de queratitis infeccionas que consultaron en un periodo de 12 meses a la fundación oftalmológica de Santander, Bucaramanga, Colombia, encontrando que Staphyloccocus cuagulasa negativo fue el principal microorganismo aislado $(28,6 \%)$, mientras que Acanthamoeba spp represento el $25 \%$ y las infecciones polimicrobiana el $23,3 \%$ de los casos. Llama la atención la elevada frecuencia de Acanthamoeba spp en esta serie de casos comparada con la reportadas en otros países (0,3-1\%). Por otro lado, no hay reportes de tratamiento con PHMB o clorohexidina (29).

Este caso se constituye en el primer caso de QA diagnosticado en el Quindío tanto por método de microscopia óptica directa como por cultivo en células Vero, con un tratamiento exitoso a base de PMHB y digluconato de clorhexidina el cual se suministró durante 12 meses, como lo descrito en otros casos reportados en la literatura. Igualmente es de resaltar la inespecificidad de los síntomas presentados por la paciente y la facilidad del diagnostico microbiológico mediante el examen microscópico directo del exudado o raspado corneal (30-32). Finalmente es importante educar a los usuarios de los lentes de contacto blandos para que realicen una adecuada limpieza y almacenamiento de los mismo, pues como se pudo ver en este caso la queratitis se asoció con el uso de este tipo de lentes, lo cual ha sido también reportado en la mayoría de los casos publicadas $(12,13,26-28)$.

\section{AGRADECIMIENTOS}

A la Doctora Alejandra de La Torre del Centro de Investigaciones Biomédicas de la Universidad del Quindío por su asistencia en el manejo del presente caso.

\section{BIBLIOGRAFÍA}

(1) Botero D, Restrepo M. Parasitosis Humanas. 4ta edición. Editorial Corporación para Investigaciones Biológicas .pp:288-294. Medellín.2003.

(2) Quijano B., López M., Moncada L.,Moreno. C. El conejo como modelo animal para la reproducción de la infección corneal por Acanthamoeba polyphaga. Infectio 2006;10: 79-84.

(3) Cabral-Marciano F and Cabral G. Acanthamoeba spp. as Agents of Disease in Humans. Clinical Microbiology Reviews. 2003;16:273-307.

(4) Latapie LB, Cremona G, Carrasco MA, Molina VE, Bozzini JP, Mariano ML . Queratitis inflamatoria por Acanthamoeba ssp. Analisis estructural por microscopia electrónica de transmisión. Parasitol Latinoam.2003, 58:159-165.

(5) Stehr-Green JK, Bailey TM, Visvesvara GS. The epidemiology of Acanthamoeba keratitis in the United States. Am J Ophthalmol 1989; 107: 331-36.

(6) Nagington J, Watson PG, Playfair TJ, et al. Amoebic infection of the eye. Lancet 1974; 2: 1537-40.

(7) Visvesvara G. S., Jones D. B., Robinson N. M. Isolation, Identification, and Biological Characterization of Acanthamoeba Polyphaga from a Human Eye.Am. J. Trop. Med. Hyg. 1975; 24: 784-90.

(8) Zheng X, Uno T, Goto T, Zhang W, Hill JM. and Ohashi Y. Pathogenic Acanthamoeba Induces Apoptosis of Human Corneal Epithelial Cells. Jpn J Ophthalmol 2004;48:23-29. 
(9) Bottone EJ, Madayag RM, Qureshi N. Acanthamoeba Keratitis: Synergy between amebic and bacterial cocontaminansts in contact lens care systems as a prelude to infection. J.Clin.Microbiol.1992;30:2447-50.

(10) Lee J.S., Hahn T.W., Choi S.H., Yu H.S., Lee JE. Acanthamoeba keratitis related to cosmetic contact lenses. Clinical \& Experimental Ophthalmology. 2007; 35: 775-77.

(11) Tzanetou K., Miltsakakis D., Droutsas D., Alimisi S., Petropoulou D ., Ganteris G et al. Acanthamoeba Keratitis and Contact Lens Disinfecting Solutions. Ophthalmologica 2006;220:238-241.

(12) Teramoto S.A., Tsuru C.L. QUERATITIS POR ACANTHAMOEBA EN USUARIOS DE LENTES DE CONTACTO: Revisión Revista de Posgrado de la Via Cátedra de Medicina.2003;123:18-

21.En:http://med.unne.edu.ar/revista/revista123/queratitis.htm.fecha de consulta: 21de abril de 2008.

(13) Pérez-Irezábal J, Isasa P, Barrón J, Martinez I. Queratitis por Acanthamoeba. En : http://www.seimc.org/control/revi Para/Acantha.htm. consultado el 21 de abril de 2008.

(14) Pérez-Santonja JJ, Kilvington S, Hughes R, Tufail A, Matheson M, Dart JK. Persistently culture positive Acanthamoeba keratitis: in vivo resistance and in vitro sensitivity. Ophthalmology.2003;110:1593-600.

(15) Manikandan P, Bhaskar M, Revathy R, John RK, Narendran V, Panneerselvam K. Acanthamoeba keratitis- A six year epidemiological review from a tertiary care eye hospital in south India. Indian Journal of Medical Microbiology.2004; 22:226-30.

(16) Bharathi J, Srinivasan M, Ramakrrishnan R, Meenakshi R, Padmavathy S, Lalitha PN. A study of spectrum of Acanthamoeba keratitis: A three-year study at a teriary eye care referral centre in South India.Indian J Ophthalmol.2007;55:37-42.

(17) Azuara-Blanco A, Sadiq AS, Hussain M, Lloyd JH, Dua HS. Successful medical treatment of Acanthamoeba keratitis. Int Ophthalmol. 1998;21:223-27.

(18) D'Aversa G, Stern GA, Driebe WT Jr. Diagnosis and successful medical treatment of Acanthamoeba keratitis. Arch Ophthalmol. 1995;113:1120-3.

(19) Duguid IG, Dart JK, Morlet N, Allan BD, Matheson M, Ficker L, Tuft S.Outcome of Acanthamoeba keratitis treated with polyhexamethyl biguanide and propamidine. Ophthalmology. 1997;104:1587-92.

(20) Donoso R, Mura JJ, López M. Acanthamoeba keratitis treated with propamidine and polyhexamethyl biguanide (PHMB). Rev Med Chil. 2002;130:396-401.

(21) Varga JH, Wolf TC, Jensen HG, Parmley VC, Rowsey JJ.Combined treatment of Acanthamoeba keratitis with propamidine, neomycin, and polyhexamethylene biguanide. Am J Ophthalmol.1993;115:466-70.

(22) Lim N, Goh D, Bunce C, Xing W, Fraenkel G, Poole TR, Ficker L.Comparison of polyhexamethylene biguanide and chlorhexidine as monotherapy agents in the treatment of Acanthamoeba keratitis. Am J Ophthalmol. 2008;145:130-5.

(23) Murdoch D, Gray TB, Cursons R, Parr D. Acanthamoeba keratitis in New Zealand, including two cases with in vivo resistance to polyhexamethylene biguanide. Aust N ZJ Ophthalmol.1998;26:231-6.

(24) Park DH, Palay DA, Daya SM, Stulting RD, Krachmer JH, Holland EJ.The role of topical corticosteroids in the management of Acanthamoeba keratitis. Cornea. 1997;16:277-83.

(25)Vaquero-Ruano M, Encinas-Martin JL, Ramon y Cajal S, Cajigal Morales C.Queratitis por Acanthamoeba, posible resistencia a dibromo propamidil isetionato.en: http://www.mpsp.org/mpsp/Documentos/Desinfec/antisep.htm. Fecha de consulta 26 de mayo de 2008.

(26)O'Day DM, Head WS.Advances in the management of keratomycosis and Acanthamoeba keratitis. Cornea. 2000;19:681-7.

(27)Shi WY, Gao H, Li SW, Wang FH, Xie LX.Clinical study of the treatment of Acanthamoeba keratitis by penetrating keratoplasty. Zhonghua Yan Ke Za Zhi. 2004 ;40:750-4.

(28) Rosero L, Delatorre A. Manejo quirúrgico de ulceras micóticas cornéales. Franja ocular .2001; 2: 5-7.

(29) De la torre A., Nuñez M.X.,Blanco C. Guias de manejo de las queratitis infecciosas bacterianas. Hospital Universitario del Valle, enero-julio 2002. Colomb. Med. 2003; 34: 132-36.

(30) Güerri N, Alías E, Borque E, Díaz S, Sánchez A, Brito C , Honrubia F. Queratopatía por acanthamoeba en portadores de lentes de contacto. a propósito de tres casos.Rev. Esp. Contact. 2006; 13:67-72.

(31)Leyre López L, de Fernando S, Gaztelurrutia L, Vilar B, Pérez-Irezábal J, Barrón J. Queratitis por Acanthamoeba spp.: presentación de diez casos. Enferm Infecc Microbiol Clin 2000; 18: 229-33.

(32) Ramirez- Molas L, Kang H, Ayala R. Queratitis por Acanthamoeba sp. Reporte de caso. An. Fac. Cienc. Méd (Asunción. 2005;.38:44-47. 